OPEN ACCESS

Edited by: Fabrizio Carta,

University of Florence, Italy

Reviewed by:

Sriram Sundaravel,

Albert Einstein College of Medicine,

United States

Vivek Sharma,

Birla Institute of Technology and

Science, India

*Correspondence: Huanjie Yang

yanghj@hit.edu.cn

Specialty section:

This article was submitted to

Cancer Molecular Targets

and Therapeutics,

a section of the journal

Frontiers in Oncology

Received: 13 July 2021 Accepted: 18 October 2021 Published: 03 November 2021

Citation:

Mei Y, Li K, Zhang Z, Li M, Yang H, Wang $H$, Huang $X$, Li $X$, Shi S and Yang $H$ (2021) miR-33b-3p Acts as a

Tumor Suppressor by Targeting DOCK4 in Prostate Cancer.

Front. Oncol. 11:740452. doi: 10.3389/fonc.2021.740452

\section{miR-33b-3p Acts as a Tumor Suppressor by Targeting DOCK4 in Prostate Cancer}

\author{
Yu Mei ${ }^{1}$, Kai $L i^{1}$, Zhicheng Zhang ${ }^{2}$, Mengmeng $\mathrm{Li}^{1}$, Hong Yang ${ }^{2}$, Hui Wang ${ }^{1}$, \\ Xuemei Huang ${ }^{1}$, Xinyuan $\mathrm{Li}^{1}$, Shuhua Shi ${ }^{1}$ and Huanjie Yang ${ }^{1 \star}$ \\ ${ }^{1}$ School of Life Science and Technology, Harbin Institute of Technology, Harbin, China, ${ }^{2}$ Fourth Affiliated Hospital of Harbin \\ Medical University, Harbin, China
}

Despite that androgen-deprivation therapy results in long-lasting responses, the disease inevitably progresses to metastatic castration-resistant prostate cancer. In this study, we identified miR-33b-3p as a tumor suppressor in prostate cancer. miR-33b-3p was significantly reduced in prostate cancer tissues, and the low expression of miR-33b-3p was correlated with poor overall survival of prostate cancer patients. Overexpression of miR-33b-3p inhibited both migration and invasion of highly metastatic prostate cancer cells whereas inhibition of miR-33b-3p promoted those processes in lowly metastatic cells. The in vivo results demonstrate that miR-33b-3p suppresses metastasis of tail vein inoculated prostate cancer cells to lung and lymph nodes in mice. DOCK4 was validated as the direct target of miR-33b-3p. miR-33b-3p decreased the expression of DOCK4 and restoration of DOCK 4 could rescue miR-33b-3p inhibition on cell migration and invasion. Moreover, downregulation of miR-33b-3p was induced by bortezomib, the clinically used proteasome inhibitor, and overexpression of miR-33b-3p enhanced the insufficient inhibition of bortezomib on migration and invasion as well as metastasis of prostate cancer cells. In summary, our findings demonstrate that miR-33b-3p suppresses metastasis by targeting DOCK4 in prostate cancer. Our results suggest that enhancing miR-33b-3p expression may provide a promising therapeutic strategy for overcoming that proteasome inhibitor's poor efficacy against metastatic prostate cancer.

Keywords: miR-33b-3p, DOCK4, metastasis, prostate cancer, proteasome inhibitor

\section{INTRODUCTION}

Prostate cancer is the most common malignancies and the second leading cause of cancerassociated mortality in men (1). Despite that androgen-deprivation therapy results in longlasting responses, the disease inevitably progresses to metastatic castration-resistant prostate cancer (mCRPC) that is associated with poor prognosis $(2,3)$. The proteasome is a validated 
target for cancer therapy. The first-in-class proteasome inhibitor bortezomib demonstrates great success against multiple myeloma (4). However, limited efficacy of bortezomib alone was observed in solid tumors including prostate cancer. As a combination regimen, bortezomib was found not able to improve prostate cancer patients' response to docetaxel, a standard treatment for mCRPC $(5,6)$.

MicroRNAs (miRNAs) are a conserved class of small noncoding RNAs that have been recognized as key regulators of various cellular processes at post-transcriptional level $(7,8)$. Dysregulation of miRNAs has been demonstrated to broadly associate with tumor invasion and angiogenesis as well as drug resistance (7-11). Proteasome inhibitor leads to alteration in not only the proteins but also the miRNAs $(12,13)$. Our previous studies showed that miR-33b-3p was downregulated in prostate cancer in response to proteasome inhibition (14). It drew our attention that miR-33b-5p was predominantly upregulated in multiple myeloma post treatment with proteasome inhibitor $(15,16)$. Both arms of the miRNAs can be expressed as a mature form in vivo $(17,18)$. In some cases, they have similar function while in some cases; they work opposite (19). It has been shown that miR-33b-5p functions as tumor suppressor in prostate cancer (20). miR-33b-3p also functions as tumor suppressor in some type of cancers $(21,22)$. Thus, we speculated that miR-33b-3p might function as a tumor suppressor in prostate cancer, which downregulation in response to bortezomib might contribute to insufficient efficacy of targeting the proteasome in prostate cancer.

In the present study, we found that miR-33b-3p was the dominant mature form in prostate cancer. Elevated miR-33b-3p significantly reduced the metastasis of prostate cancer cells by targeting DOCK4. Moreover, downregulation of miR-33b-3p post bortezomib treatment was associated the insufficient inhibition on prostate cancer cells migration and invasion.

\section{MATERIALS AND METHODS}

\section{Cell Culture}

Prostate and lung cancer cells were cultured in RPMI 1640 medium with $10 \%$ fetal bovine serum (FBS, Gibco-BRL, Grand Island, NY, USA) and breast cancer cells were cultured in DMEM medium with $10 \%$ FBS in a humid atmosphere containing $5 \% \mathrm{CO}_{2}$ at $37^{\circ} \mathrm{C}$.

\section{Primers and Oligos}

Primers were synthesized by Comate Biosciences (Jilin, China). The mimic and inhibitor of miR-33b-3p were obtained from Ribio (Guangzhou, China). The primer and oligo sequences are listed in Table 1.

\section{RT-qPCR}

Total RNAs were isolated from culture cells or frozen prostate cancer tissues using Trizol (Invitrogen, Carlsbad, CA, USA) and reverse-transcribed (RT) into cDNA (500 ng total RNA) using ReverTra Ace (Toyobo, Kita-Ku, Osaka, Japan). Quantitative realtime PCR (RT-qPCR) was performed using SYBR Premix Ex Taq (Takara, Otsu, Shiga, Japan). The efficiency of primer for both arms of miR-33b (E) was determined from the slope of the curve obtained with the serially diluted standards $\left(10^{8}-10^{5}\right.$ gene copies per qPCR reaction), as $\mathrm{E}=10^{(-1 / \text { slope })}(23)$. The slopes of the calibration curves were calculated from the plot of the log base 10 of initial gene copy number versus corresponding threshold cycles (Ct values) and used to determine the number of gene copies in each sample. Microarray gene profiling datasets of GEO database was used to compare the expressions of both arms of miR-33b in normal prostate tissues and prostate cancer samples (http://www.ncbi.nlm.nih.gov/geo, GEO accession: GSE64318).

\section{Plasmid and Transfection}

The wild type of 3' UTR of DOCK4 was amplified by PCR and cloned into the SpeI/MluI (Takara, Otsu, Shiga, Japan) sites of pMIR-REPORT vector (Promega, Madison, WI, USA). The mutated 3' UTR of DOCK4 was amplified using two-stepwise PCR and cloned into the same sites of pMIR-REPORT vector. The full length of DOCK4 was amplified by overlapping PCR using three pairs of primers and cloned into the XhoI/NotI (Takara, Otsu, Shiga, Japan) sites of pIRM-3xHA (HA) vector (BCCM, Zwijnaarde, Gent, Belgium). The primer sequences are listed in Table 1. All these constructed plasmids were confirmed by sequencing. Transient transfection of miRNA or plasmids were mediated by Lipofectamine 3000 (Invitrogen, Carlsbad, CA, USA) reagent following the manufacturer's protocol.

\section{Stable Cell Lines}

pLKO.1-puro plasmid (Addgene, Watertown, MA, USA) was used for miR-33b-3p overexpression. Sense and antisense of miRNA oligos listed in Table $\mathbf{1}$ were annealed and inserted into the Age I/EcoRI (Takara, Otsu, Shiga, Japan) digested pLKO.1-puro vector. Stable cell lines were established through lentiviral transduction. Briefly, lentiviral packaging mix (VSV-G plasmid and Gag-Pol plasmid) and constructed pLKO.1 plasmid were co-transfected into HEK293T cells. The supernatants containing lentiviruses were collected, filtered, and added into indicated cells. After $48 \mathrm{~h}$ of incubation with lentiviruses, the transduced cells were selected with puromycin (Santa Cruz, TX, USA). The knockdown efficiency or overexpression level was confirmed by RT-qPCR. Cells stably expressing firefly luciferase were confirmed by measuring the luciferase activity.

\section{MTT Assay}

LNCaP and PC3 prostate cancer cells were transfected with miR33b-3p mimic, inhibitor or either negative control for $24 \mathrm{~h}$. After transfection, the cells $\left(1 \times 10^{4} /\right.$ well $)$ were planted in 96 -well plates and cultured for 72 or $96 \mathrm{~h}$. The 3-(4, 5-dimethylthiazolyl-2)-2, 5 -diphenyltetrazolium bromide (MTT) ( $5 \mathrm{mg} / \mathrm{mL}$; Sigma, MO, USA) was added into each well, and dissolved in $150 \mu \mathrm{L}$ DMSO (Sigma) after $4 \mathrm{~h}$ incubation. The absorbance was measured at a wavelength of $490 \mathrm{~nm}$ by using a microplate spectrophotometer (Bio-Rad, CA, USA). 
TABLE 1 | Oligos used for PCR and vector construction.

\begin{tabular}{|c|c|}
\hline Reverse Transcription primers & Sequences (5'-3') \\
\hline $\operatorname{miR}-33 b-3 p$ & CTCAACTGGTGTCGTGGAGTCGGCAATTCAGTTGAGGG GCTGCA \\
\hline miR-33b-5p & CTCAACTGGTGTCGTGGAGTCGGCAATTCAGTTGAGGC AATGCA \\
\hline qRT-PCR primers & Sequences $\left(5^{\prime}-3^{\prime}\right)$ \\
\hline \multirow[t]{2}{*}{$\operatorname{miR}-33 b-3 p$} & Forward: ACACTCCAGCTGGGCAGTGCCTCGGCAGTG \\
\hline & Reverse: TGGTGTCGTGGAGTCG \\
\hline \multirow[t]{2}{*}{ miR-33b-5p } & Forward: ACACTCCAGCTGGGGTGCATTGCTGTTG \\
\hline & Reverse: TGGTGTCGTGGAGTCG \\
\hline \multirow[t]{2}{*}{ U6 } & Forward: CGCTTCGGCAGCACATATACTA \\
\hline & Reverse: CGCTTCACGAATTGGGTGTCA \\
\hline \multirow[t]{2}{*}{ DOCK4 } & Forward: GACCCACACACAGACTGCTTCA \\
\hline & Reverse: GAGAGGGGGTGAAAGACTGC \\
\hline \multirow[t]{2}{*}{ GAPDH } & Forward: TGCACCACCAAACTGCTTAGC \\
\hline & Reverse: GGCATGGACTGTGGTCATGAG \\
\hline Plasmids construction Primers & Sequences (5'-3') \\
\hline \multirow[t]{2}{*}{ pMIR-WT-3' UTR } & Forward: GACTAGTITTCTATGTACCTGCGATGC \\
\hline & Reverse: CGACGCGTACGGTGTCAGGGTAGTAAGG \\
\hline \multirow[t]{2}{*}{ pMIR-MUT-3' UTR-1 } & Forward: TाTAGTGTCGTCGCGTTAATAACTT \\
\hline & Reverse: CGACGCGTACGGTGTCAGGGTAGTAAGG \\
\hline \multirow[t]{2}{*}{ pMIR-MUT-3' UTR-2 } & Forward: TAAGTTATTAACGCGACGACACTAA \\
\hline & Reverse: TAGTTGACCGCTTGAAGTCT \\
\hline \multirow[t]{2}{*}{ HA-DOCK4-1 } & Forward: AAACTCGAGATGTGGATACCTACGGAGCACGAG \\
\hline & Reverse: GCATCAGAGGGACAAAAGAAAACC \\
\hline \multirow[t]{2}{*}{ HA-DOCK4-2 } & Forward: CGATGTTCATTGTAGACAGTAGTG \\
\hline & Reverse: CCATTAGACGAGTTACAGTAGCA \\
\hline \multirow[t]{2}{*}{ HA-DOCK4-3 } & Forward: AGAAAAAGGTGTTAGAAAAGTATGG \\
\hline & Reverse: AAAGCGGCCGCTTATAACTGAGAGACCTTGCGGGGC \\
\hline miRNA construction oligos & Sequences (5'-3') \\
\hline \multirow[t]{2}{*}{ pLKO.1-miR-33b-3p } & Sense: CCGGCAGTGCCTCGGCAGTGCAGCCCCTCGAGGGGCT GCACTGCCGAGGCACTGTIITG \\
\hline & Antisense: AATTCAAAAACAGTGCCTCGGCAGTGCAGCCСCTCGAG GGGCTGCACTGCCGAGGCACTG \\
\hline
\end{tabular}

\section{Western Blotting}

Cells were lysed radioimmunoprecipitation assay (RIPA) buffer (10 mM Tris-HCl, $150 \mathrm{mM} \mathrm{NaCl}, 0.1 \%$ SDS, $0.8 \%$ Triton X-100) containing $1 \times$ protease inhibitor cocktail (Roche, Mannheim, Germany) (Roche, Mannheim, Germany). Western blotting was performed as described previously (24). The primary antibodies were mouse anti-GAPDH (KC-5G4) (Kangchen, Shanghai, China), $\beta$-actin (Santa Cruz, CA, USA) and DOCK4 (Proteintech, Wuhan, China). The secondary antibodies were from Cell Signaling Technology (MA, USA).

\section{Wound Healing Assay}

Cells $\left(3 \times 10^{5} /\right.$ well $)$ were planted in 6-well plate. For starvation, cells were cultured in the serum-free medium for $24 \mathrm{~h}$. The scratch was made by using the $200 \mu \mathrm{L}$ plastic pipette tip. The scratched cells were washed with $1 \times$ PBS to remove debris. The scratches were photographed after $24 \mathrm{~h}$ and $48 \mathrm{~h}$ incubation.

\section{Transwell Assay}

Cell migration and invasion assays were performed using Transwell chambers with polycarbonate filter ( $8 \mu \mathrm{m}$ pore) (Corning Costar, Cambridge, MA, USA). Cells were trypsinized and resuspended in serum-free medium. Cell suspensions $\left(4 \times 10^{4}\right.$ cells in $\left.150 \mu \mathrm{L}\right)$ were seeded in the upper chamber, and $500 \mu \mathrm{L}$ medium containing $20 \%$ FBS was added to the lower chamber. Cells were incubated at $37^{\circ} \mathrm{C}$ for $48 \mathrm{~h}$. Cells remaining on the surface of the upper chamber were removed by a cotton swab while cells migrated into the lower chamber were fixed with methanol and stained with $0.5 \%$ crystal violet (Sinopharm Chemical Reagent, Shanghai, China).

For cell invasion assay, Transwell filters were pre-coated with $50 \mu \mathrm{L}$ Matrigel (BD Biosciences, Franklin Lakes, NY, USA) at working concentration $(0.3 \mathrm{mg} / \mathrm{mL})$ in serum-free medium. The assay was performed following the migration assay protocol. The migrated and invasive cells were counted under microscope and quantified by Image J software (NIH, Bethesda, MD, USA).

\section{Dual Luciferase Reporter Assay}

Prostate cancer cells $\left(1 \times 10^{5}\right.$ cells/well $)$ were grown in 24 -well plate. The pcDNA3.1-miR-33b $(0.3 \mu \mathrm{g})$ was co-transfected with pMIR-WT-3' UTR $(0.3 \mu \mathrm{g})$ or pMIR-MUT-3' UTR of DOCK4 $(0.3 \mu \mathrm{g})$ in the presence of pRLSV40 $(0.03 \mu \mathrm{g})$ into cells for $48 \mathrm{~h}$. Luciferase activities were assessed by Dual-Luciferase Reporter Assay System (Promega, Fitchburg, WI, USA). Renilla luciferase activity was normalized to the firefly luciferase.

\section{RNA-Seq Analysis}

Total RNA was extracted by Trizol (Ambion, CA, USA). Library preparation and transcriptome sequencing were performed by Novogene (Beijing, China). The library preparations were sequenced on an Illumina Novaseq platform and $150 \mathrm{bp}$ paired-end reads were generated. The raw data of RNA-seq was deposited to the Gene Expression Omnibus (GEO) (GEO accession number: GSE183428). 
Differential expression analysis of two groups (three biological replicates per group) was performed using the DESeq2 R package (1.20.0). The resulting P-value was adjusted using the Benjamini and Hochberg's approach for controlling the false discovery rate. Genes with an adjusted $P<0.05$ found by DESeq2 were assigned as differentially expressed.

Gene Ontology (GO) enrichment analysis of differentially expressed genes was implemented by the clusterProfiler $\mathrm{R}$ package, in which gene length bias was corrected. GO terms with corrected $\mathrm{P}<0.05$ were considered significantly enriched by differential expressed genes.

\section{In Vivo Study}

All studies in animals were performed according to protocol approved by the Animal Care and Use Committee of Harbin Institute of Technology. BALB/c nude mice aged at 4 weeks were obtained from Beijing Vital River Laboratory Animal Technology Co., Ltd. and housed in a specific pathogen-free environment. The mice were inoculated with PC-3M-1E8luciferase cells $\left(1.5 \times 10^{6}\right)$ through tail vein, and luminescent signal was monitored every week. After 7 weeks of inoculation, the mice were sacrificed. The removed lung, liver, lymph nodes, and tumor tissues were fixed with $4 \%$ paraformaldehyde.

For the bortezomib treatment experiment, miR-33b-3poverexpressing and control PC-3M-1E8-luciferase cells were injected into the mice through tail vein. After 30-days inoculation, each group was administrated with bortezomib (LC Labtoratories, MA, USA) ( $\mathrm{n}=5)$ at a dose of $0.5 \mathrm{mg} / \mathrm{kg}$ body weight in $100 \mathrm{uL}$ or equal volume of PBS $(n=4)$, respectively. The drug was administered by intraperitoneal injection for four weeks, twice a week. Then, the mice were sacrificed except for 2 mice from bortezomib treated control group which were died after two weeks of treatment. The removed lungs were fixed with $4 \%$ paraformaldehyde. The samples from both experiments were embedded in paraffin and cut at 10 $\mu \mathrm{M}$. Sections were deparaffinized and stained with hematoxylin and eosin (H\&E), then mounted for microscope observation.

\section{Overall Survival Analysis}

The overall survival curves of patients from TCGA was plotted by Kaplan-Meier method and the log-rank test. Patients were divided into high and low groups of miR-33b-3p expression according to the median value.

\section{Statistical Analysis}

Microsoft Excel software was used for statistical analysis. Results for continuous variables are presented as means \pm SD unless stated otherwise. For two-group comparison, two-tailed Student's $t$-test was used.

\section{RESULTS}

\section{Downregulation of miR-33b-3p in Prostate Cancer Cells}

Our previous array analysis showed that the $3 \mathrm{p}$ arm of miR-33b was downregulated in response to proteasome inhibition by both the authentic inhibitor bortezomib and celastrol with proteasome inhibitory effect in prostate cancer $(14,25)$. As both arms of miRNAs can be processed into mature form depending on different cell context $(17,18)$, we determined the expression levels of the two arms of miR-33b in prostate cancer cell lines. Firstly, the efficiency of primers for both arms of miR$33 \mathrm{~b}$ was evaluated, showing that the primers for miR-33b-3p and miR-33b-5p had equal amplification efficiency (Figure 1A). Then the copy number of per sample (500 ng reverse transcribed RNA) was calculated based on the Ct values by using the calibration curve of each arm of miR-33b. The results showed that miR-33b-3p was the major mature form in prostate cancer cell lines, while miR-33b-5p was almost undetected (Figure 1B). On the contrary, miR-33b-5p was the major form in breast and lung cancer cell lines (Figure 1C). Analysis of clinical samples indicated that both arms of miR-33b can be processed to mature forms; however, miR-33b-3p was the major isoform in normal prostate tissues as well as tumor samples (Figure 1D). In addition, miR-33b-3p level was downregulated in tumor tissues compared with the normal counterpart while no significant difference was observed in terms of miR-33b-5p level (Figure 1D). The overall survival analysis using the KaplanMeier curves demonstrated that prostate cancer patients with high level of miR-33b-3p exhibited significantly better survival (logrank $p=0.024414$ ) (Figure 1E). These results suggest that miR-33b-3p might act as tumor suppressor in prostate cancer.

\section{miR-33b-3p Inhibits Prostate Cancer Cells Proliferation}

To investigate the tumor suppressive role of miR-33b-3p in prostate cancer cells, miR-33b-3p mimic or inhibitor were transfected into the prostate cancer cells, and the efficacy of transfection was determined by RT-qPCR (Figures 2A-D). MTT assays showed that compared with the group transfected with the mimic control, transfection with miR-33b-3p mimic greatly reduced cell numbers in androgen receptor (AR) positive LNCaP and AR negative PC3 cells (Figures 2E, F). Conversely, transfection with miR-33b-3p inhibitor led to enhanced cell proliferation in LNCaP and PC3 cells (Figures 2E, F). Cell viability was not affected by altered miR-33b-3p expression levels, no matter overexpression or inhibition (Figures 2G-J).

\section{miR-33b-3p Inhibits Prostate Cancer Cells Migration and Invasion}

To investigate the role of $\mathrm{miR}-33 \mathrm{~b}-3 \mathrm{p}$ in migration and invasion of prostate cancer cells, the PC-3M-1E8 and PC-3M2B4 cell lines that are derived from PC-3M prostate cancer cells but with different metastatic potentials were used as cell models (26). We confirmed that miR-33b-3p was still the major mature form in both PC-3M-1E8 and PC-3M-2B4 cells (Figures 3A, B). In addition, miR-33b-3p level was found much lower in highly metastatic PC-3M-1E8 than that in the lowly metastatic PC-3M-2B4 cells (Figure 3C). Wound healing assay showed that PC-3M-1E8 cells with miR-33b-3p mimic transfection had slower recovery rates than control cells (Figure 3D). On the 

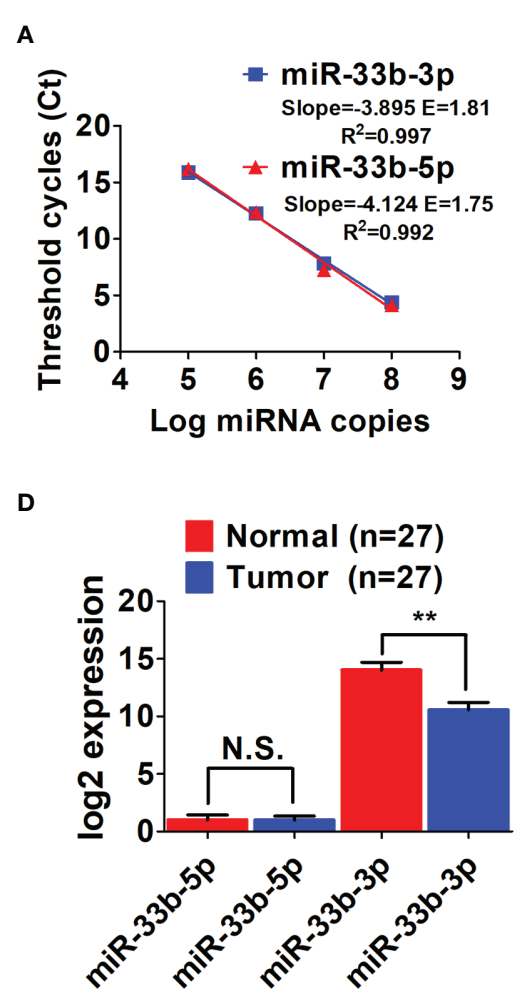

B

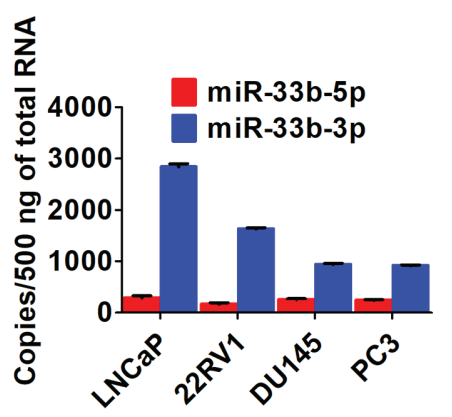

E
C

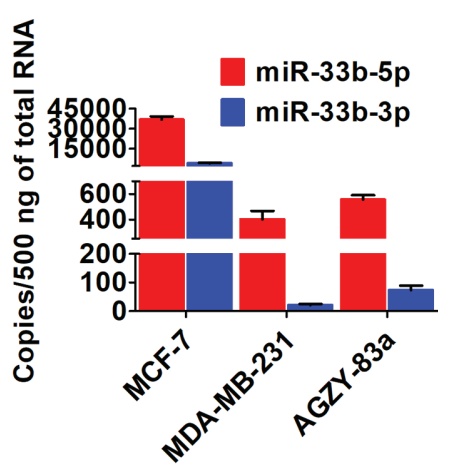

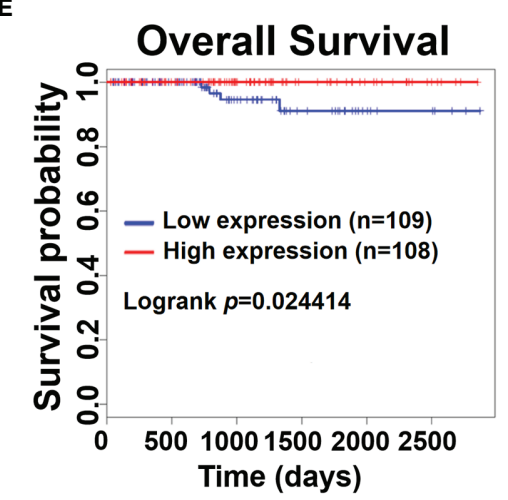

FIGURE 1 | miR-33b-3p was downregulated in prostate cancer cells. (A) Ct values versus log miRNA copies were plotted to calculate the slope. The corresponding RT-PCR primers efficiencies were calculated according to the equation: $E=10^{[-1 / s l o p e]}$. RT-qPCR analysis of both arms of miR-33b in prostate (B), breast and lung cancer cell lines (C) as well as tumor and adjacent normal tissues of prostate adenocarcinoma patients ( $n=27)$ (D). (E) Kaplan-Meier curve of overall survival of prostate adenocarcinoma patients analyzed by using TCGA. Blue and red curve represents patients with low or high expression of miR-33b-3p, respectively. Data (A-C) are representative of at least three independent experiments. N.S., not significant, ${ }^{\star \star} P<0.01$ by two-tailed Student's $t$-test.

contrary, transfection with miR-33b-3p inhibitor in PC-3M2B4 cells led to faster wound closure than that control group (Figure 3E). Transwell assays showed that overexpression of miR-33b-3p inhibited the migrative and invasive ability of highly metastatic PC-3M-1E8 cells. Conversely, transfection of miR-33b-3p inhibitor accelerated PC-3M-2B4 cells invasion and migration (Figures 3F, G). Together, these results demonstrate that miR-33b-3p can inhibit the invasion of prostate cancer cells in vitro.

\section{miR-33b-3p Inhibits Prostate Cancer Metastasis In Vivo}

To investigate whether miR-33b-3p could suppress metastasis in vivo, the luciferase reporter gene was introduced into PC-3M$1 \mathrm{E} 8$ cells to trace tumor metastasis. The PC-3M-1E8 expressing luciferase cells were transfected with pLKO.1-miR-33b-3p or pLKO.1 vector. Overexpression of miR-33b-3p was confirmed (Figure 4A), then the cells were injected into BALB/c nude mice through tail vein, and metastasis of cancer cells was monitored via D-luciferin. Luminescence imaging showed that overexpression of miR-33b-3p led to significant decrease in luminescent intensity in the whole body as well as the removed organs (Figures 4B, C). Dissection of mice organs further revealed that lung metastasis was detected in the control group but not miR-33b-3p group (Figure 4C). In addition, more lymph nodes metastasis was detected in the control group (7 luminescent lymph nodes in 3 mice) compared to miR-33b-3p group (2 luminescent lymph nodes in 2 mice) (Figure 4C). HE staining confirmed that lung metastasis was only observed in the control group (Figure 4D). Moreover, PC-3M-1E8 cells extensively metastasized to the lymph nodes and liver in the control group, whereas it was significantly suppressed in miR33b-3p group (Figure 4D). These results demonstrate that miR33b-3p exerts its anti-metastasis function in vivo. We further analyzed expression of miR-33b-3p in prostate cancer patients with different TNM stages using TCGA datasets (http://www. cbioportal.org/). The expression of miR-33b-3p had no difference between different $\mathrm{T}$ or $\mathrm{M}$ stages in prostate cancer patients (Supplementary Figures 3A, B).

\section{miR-33b-3p Targets DOCK4 to Regulate Cell Motility in Prostate Cancer}

To further elucidate the underlying molecular mechanism, three online prediction softwares (TargetScan, miRWalk and miRPathDB) were used to predict miR-33b-3p targets. Total 220 target genes were predicted through integration of the three 


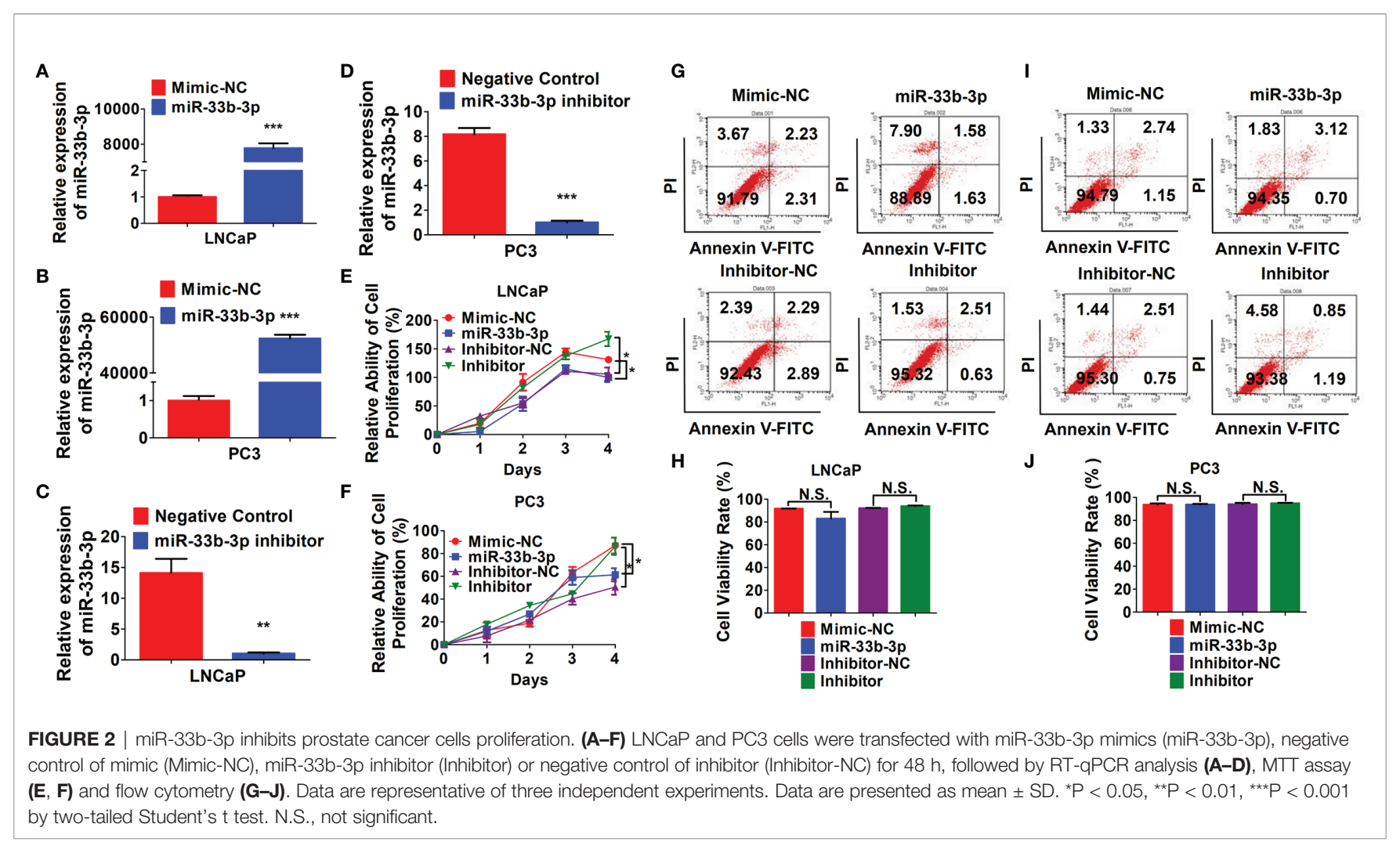

programmes (Figure 5A). The top 15 potential targets of miR-33b-3p were selected based on the confidence level (Figure 5A). Among them, the expression level of DOCK4 was the most different between normal and prostate cancer tissues (Table 2). Overexpression of miR-33b-3p led to deceased expression of DOCK4 in both mRNA and protein levels in highly metastatic PC-3M-1E8 cells, while inhibition of miR$33 \mathrm{~b}-3 \mathrm{p}$ could enhance the expression of DOCK4 in lowly metastatic PC-3M-2B4 cells (Figures 5B, C). Moreover, PC-3M-1E8 cells with low level of miR-33b-3p had high protein level of DOCK4 in relative to PC-3M-2B4 cells (Figure 5D), indicating that endogenous miR-33b-3p level had an influence on DOCK4 expression.

To determine whether miR-33b-3p directly regulated DOCK4 expression, luciferase reporters carrying the wild type or mutation of miR-33b-3p binding site in the 3' UTR of DOCK4 were constructed (Figure 5E). Dual luciferase assays showed that miR-33b-3p suppressed the luciferase activity of the reporter containing the wild type 3' UTR of DOCK4 in PC-3M-1E8 cells, and this effect was abrogated with the mutated reporter (Figure 5F). These results indicate that DOCK4 is a target of miR-33b-3p in prostate cancer cell lines. Whether miR-33b-3p exerted the motility-suppressing roles by targeting DOCK 4 in prostate cancer was determined. DOCK4 was overexpressed in PC-3M-1E8 cells (Figure 5G). Wound healing assay showed that the inhibition of wound closure caused by miR-33b-3p overexpression was restored back to control level when DOCK4 was overexpressed (Figure 5H). Consistently, DOCK4 overexpression significantly antagonized the inhibitory effects of miR-33b-3p on the migration and the invasion in PC-3M-1E8 cells (Figures 5I, J). Furthermore, overexpression of DOCK4 was observed in primary prostate tumors compared with normal tissues in TCGA dataset (Figure 5K). In addition, the expression of DOCK4 was upregulated along with the increased patient's gleason score (gleason score 6-9) (Figure 5L). These results indicate that the motility-suppressing effects of miR33b-3p on prostate cancer cells were associated with downregulation of DOCK4. Analysis of DOCK4 expression in prostate cancer patients using TCGA and UALCAN datasets (http://ualcan.path.uab.edu/index.html) showed that the level of DOCK4 had no difference between different $\mathrm{T}$ or $\mathrm{N}$ stages (Supplementary Figures 4A, B). Analysis of disease-free survival using the Gene Expression Profiling Integrative Analysis (GEPIA; http://gepia.cancer-pku.cn) showed that high expression of DOCK4 in prostate cancer tissues was associated with a shorter lifespan of patients $(\mathrm{p}=0.032)$ (Supplementary Figure 4C), indicating that high level of DOCK4 correlates with poor prognosis of prostate cancer patients.

To investigate the global changes of biological processes influenced by miR-33b-3p, we overexpressed miR-33b-3p in PC-3M-1E8 cells and performed RNA-Seq. As compared with control, enforced expression miR-33b-3p in prostate cancer cells led to high enrichment of several terms that downregulate cholesterol biosynthesis, cell proliferation and cell motility as well as angiogenesis (Figure $\mathbf{5 M}$ ). These results indicate that miR-33b-3p regulates the extensive biological processes that are 

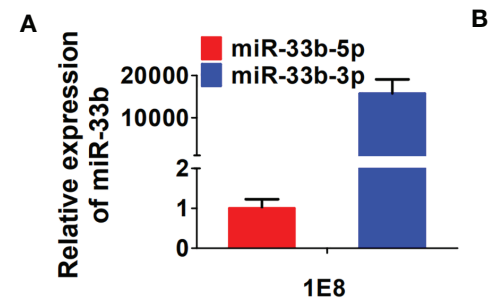

D

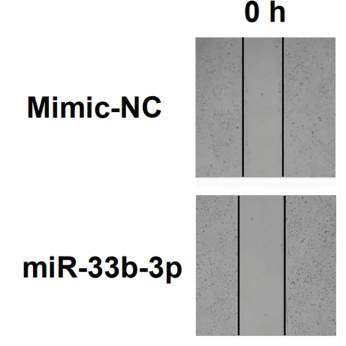

E

Inhibitor-NC

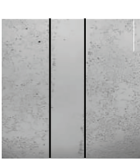

Inhibitor

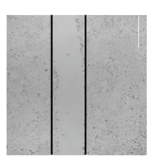

$\mathbf{F}$

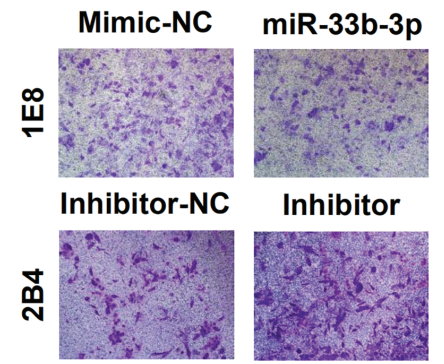

G
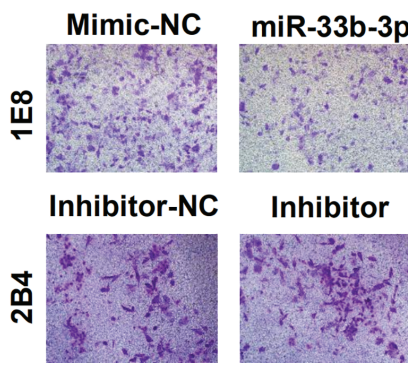

Inhibitor

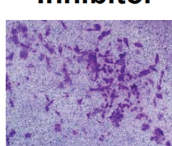

$1 E 8$

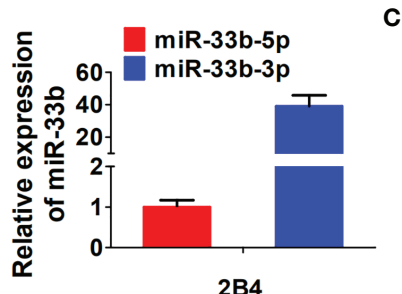

C

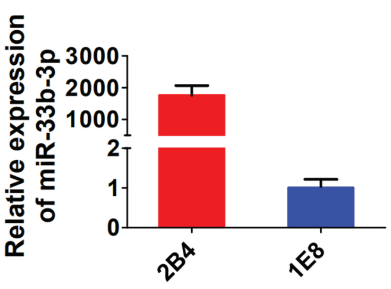

$24 \mathrm{~h}$

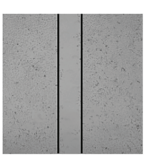

$48 \mathrm{~h}$
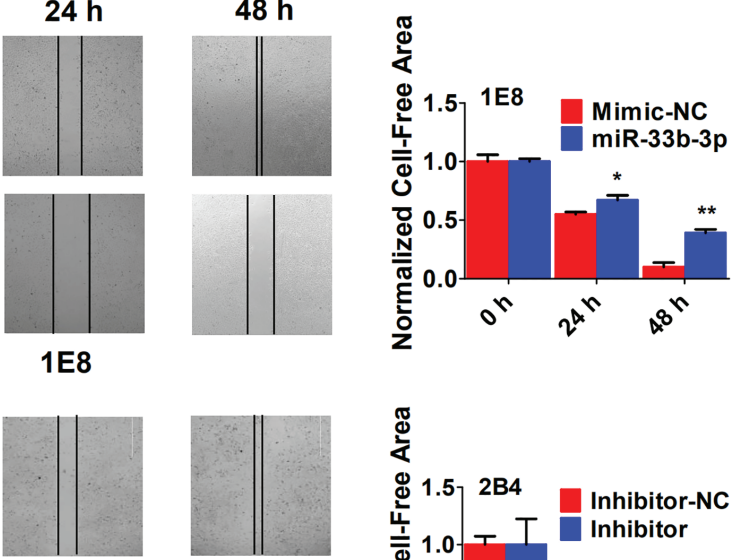

đ্்

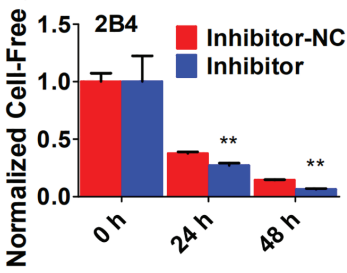

2B4
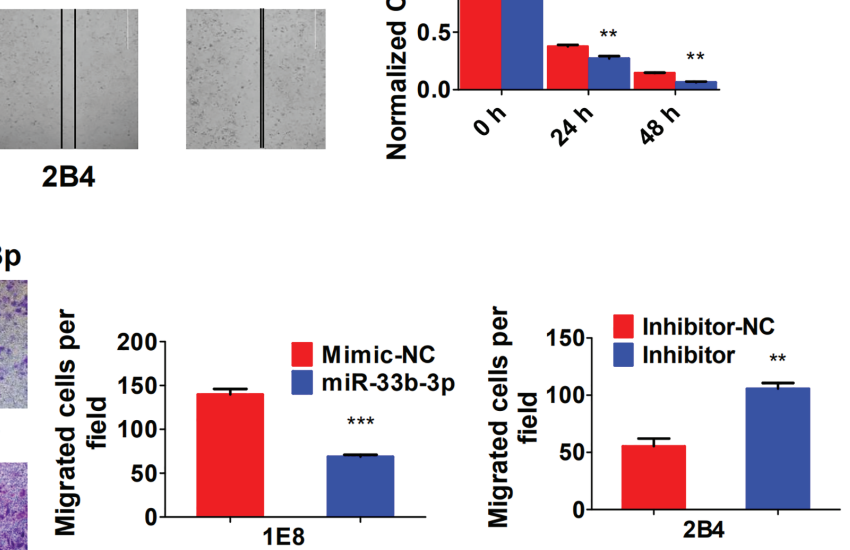

FIGURE 3 | miR-33b-3p inhibits prostate cancer cells migration and invasion in vitro. (A-C) RT-qPCR analysis in PC-3M-1E8 and PC-3M-2B4 cells with highly or lowly metastatic ability, respectively. PC-3M-1E8 or PC-3M-2B4 cells were transfected with miR-33b-3p mimic or inhibitor for 48 h, followed by wound healing (D, E) and transwell assay to examine migration $\mathbf{( F )}$ and invasion (G). Data are representative of at least three independent experiments. ${ }^{\star} P<0.05$, ${ }^{\star \star} P<0.01,{ }^{\star \star \star} P<0.001$ by two-tailed Student's $t$ test. 
A

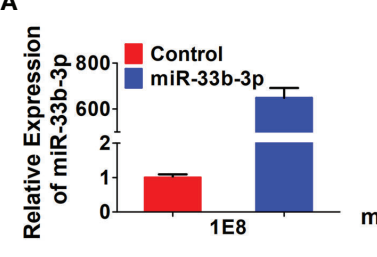

B

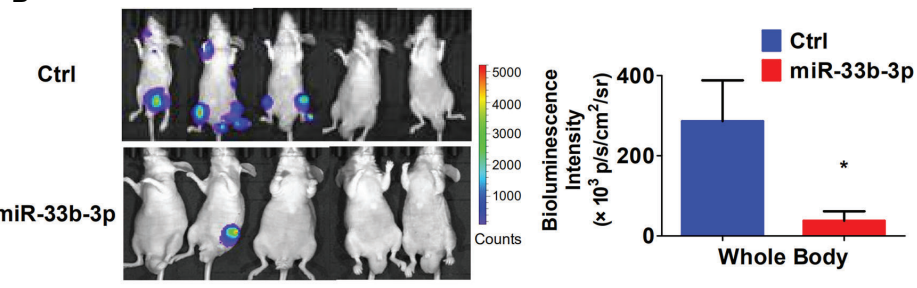

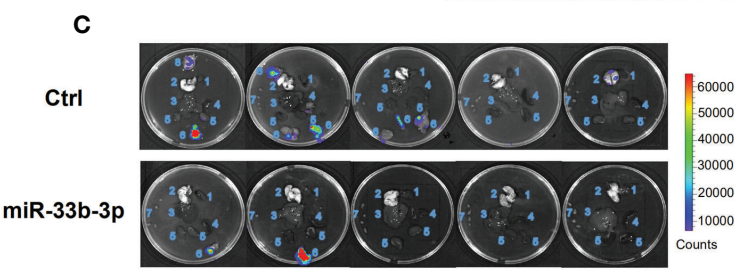

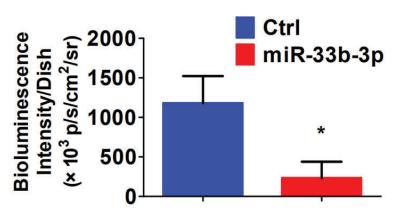

D

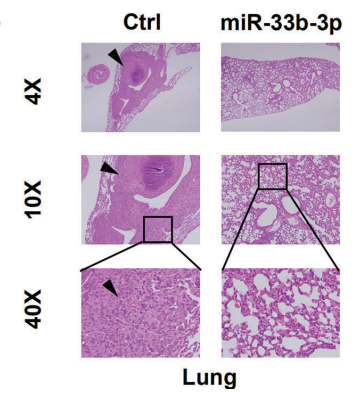

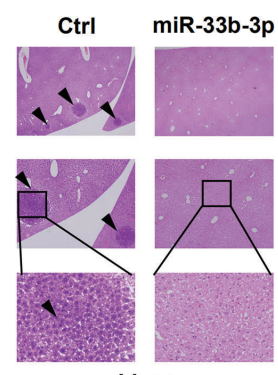

Liver
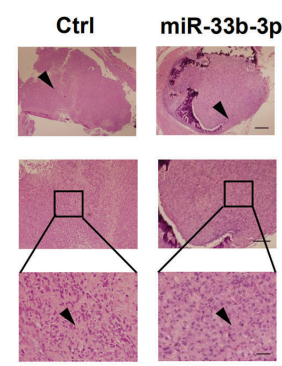

Lymph node

FIGURE 4 | miR-33b-3p inhibits prostate cancer metastasis in vivo. (A) RT-qPCR analysis in PC-3M-1E8-luciferase cells. (B, C) miR-33b-3p overexpression or control PC-3M-1E8-luciferase cells $\left(1.5 \times 10^{6}\right.$ per inoculation) were injected into BALB/c nude mice through tail vein $(n=5)$. Mice were sacrificed after 7 weeks, and bioluminescent intensity (photons/sec/cm2/steradian) of whole body (B) and removed organs (C) were detected and analyzed via D-luciferin. 1 Heart, 2 Lung, 3 Liver, 4 Spleen, 5 Kidneys, 6 Lymph nodes with luminescence signal, 7 Lymph nodes without luminescence signal, 8 Cyst. (D) H\&E staining. Arrowheads indicate cancer metastasis foci. The scale bar represents $4 \times, 10 \mathrm{~mm} ; 10 \times, 5 \mathrm{~mm}$; and 40x, $1 \mathrm{~mm}$. ${ }^{*} \mathrm{P}<0.05$.

associated with prostate cancer cells homing and growth in the metastatic sites.

\section{Overexpression of miR-33b-3p Enhances Bortezomib Effects}

Given that bortezomib demonstrated efficacy against multiple myeloma but not solid cancers, we speculated that bortezomibinduced miR-33b-3p inhibition might contribute to its inefficacy in prostate cancer. We confirmed that bortezomib inhibited miR-33b-3p expression in prostate cancer cell lines (Figure 6A). qRT-PCR indicated that the expression of primiR-33b and pre-miR-33b was also downregulated by bortezomib (Figures 6B, C). Docetaxel is the clinically used chemotherapeutics of mCRPC. As an effective agent, treatment with docetaxel increased the expression of miR-33b-3p in LNCaP cells (Figure 6D). These results suggest that the downregulation of miR-33b-3p might be associated with bortezomib's inefficacy in prostate cancer cells. Next, we determined whether bortezomib-induced miR-33b-3p inhibition might contribute to its inefficacy in prostate cancer. Based on MTT assay (Supplementary Figure 1A), non-cytotoxic dose of bortezomib was used to treat LNCaP and PC3 cells for transwell assays. Cell death assay confirmed that bortezomib did not affect cell viability in miR-33b-3p or control treated cells (Figures 6E, F). Transwell assays indicated that overexpression of miR-33b-3p (Supplementary Figures 2A, B) promoted the inhibitory effect of bortezomib on motility of prostate cancer cells (Figures 6G, H).

To further determine whether overexpression of miR-33b-3p could influence the effect of bortezomib on the metastasis of prostate cancer in vivo, miR-33b-3p-overexpressing and control PC-3M-1E8-luciferase cells were injected into the mice through tail vein. After 30-days inoculation, each group was administrated with bortezomib or equal volume of PBS, respectively. After four weeks, the mice were sacrificed. H\&E staining confirmed the development of lung metastasis in four groups (Figure 6I). The summarize of H\&E results showed that the control group receiving PBS all developed lung metastasis (4/ 4), while control group receiving bortezomib developed lung metastasis in 4/5. Again, we observed that miR-33b-3p inhibited metastasis (1/4 lung metastasis in miR-33b-3p group receiving PBS) and over-expression of miR-33b-3p could enhanced bortezomib effects on suppression of lung metastasis (2/5). This result indicates that bortezomib had limited effect on metastasis suppression in prostate cancer which can be enhanced by miR-33b-3p. 
A

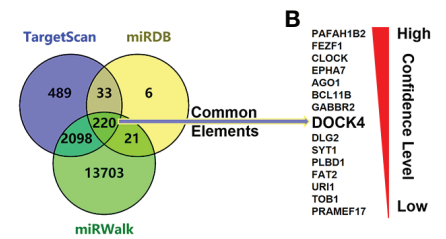

E

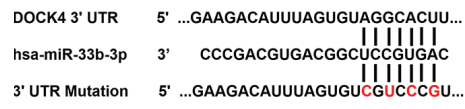

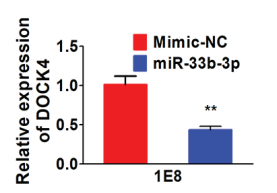

$\mathbf{F}$

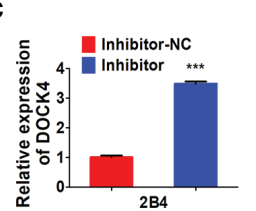

G

D

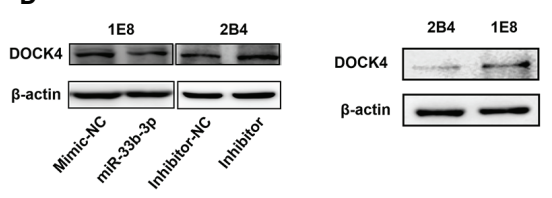
Mimic-NC + miR-33b-3p Mimic-NC + miR-33b-3p +
HA-CTRL + HA-CTRL HA-DOCK4 HA-DOCK4 HA-CTRL + HA-CTRL HA-DOCK4 HA-DOCK4

I

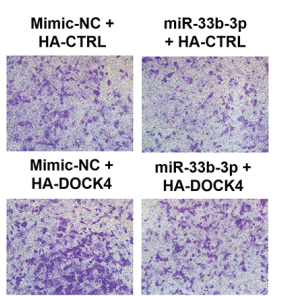

$\mathbf{K}$

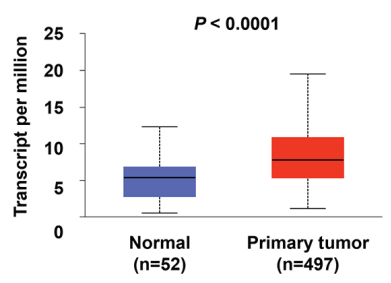

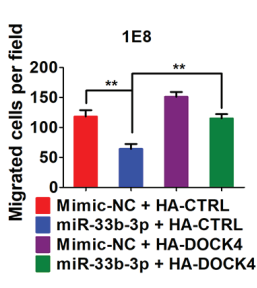

L

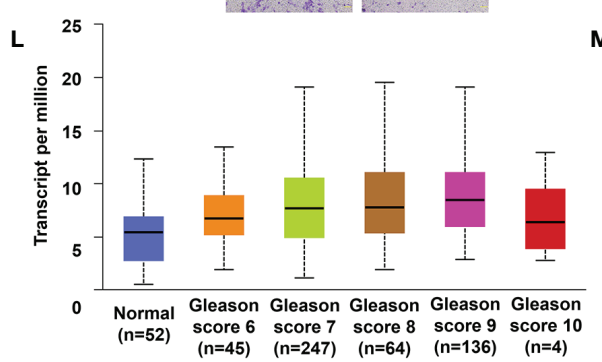

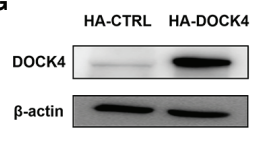

$1 E 8$

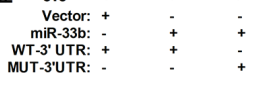

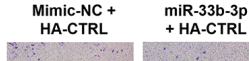
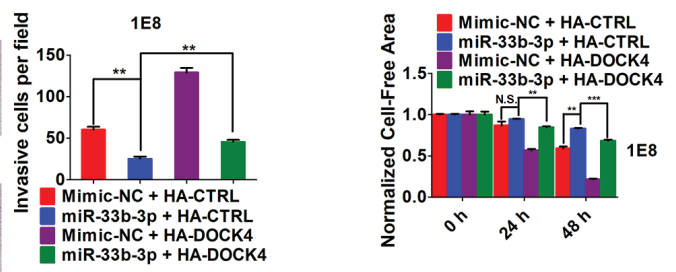

M

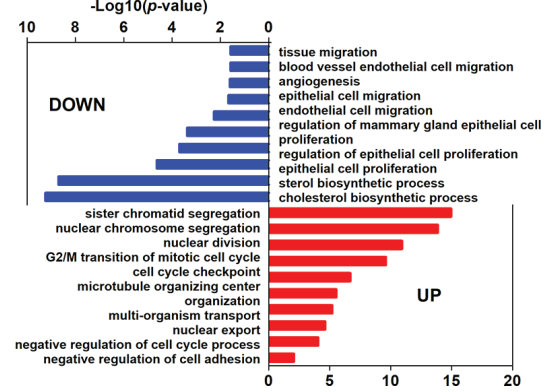

FIGURE 5 | miR-33b-3p targets DOCK4 to regulate cell motility in prostate cancer. (A) The top 15 targets of miR-33b-3p were predicted by miRPathDB, TargetScan and miRWalk. (B, C) RT-qPCR and Western blotting analysis of DOCK4 in PC-3M-1E8 and PC-3M-2B4 cells post transfection with indicated oligoes. (D) Comparison of DOCK4 level between PC-3M-1E8 and PC-3M-2B4 cells. (E, F) Luciferase reporter assay. Luciferase reporter constructs containing wild-type (WT-3'UTR) or mutation (MUT-3'UTR) in the 3' UTR of DOCK4 were generated (E) and co-transfected with pcDNA3.1-miR-33b (miR-33b) or pcDNA3.1 empty vector (Vector) for $24 \mathrm{~h}$. Luciferase activity was determined (F). (G) Western blotting analysis of DOCK4 protein level in DOCK4 overexpression (HA-DOCK4) and control (HA-CTRL) PC-3M-1E8 cells. (H-J) DOCK4 overexpression and control PC-3M-1E8 cells were transfected with mimic negative control (Mimic-NC) or miR-33b-3p mimics (miR-33b-3p) for 48 h, followed by wound healing (H) and Transwell assays (I, J). (K) The expression of DOCK4 in TCGA. (L) The expression of DOCK4 based on patient's gleason score analyzed by using TCGA. (M) Gene Ontology (GO) functional enrichment (Biological Process, BP) analysis of differentially expressed genes between the control group and miR-33b-3p overexpression group. The value -log10(p-value) > 1.5 was used as the threshold to select GO terms. Data (B, F-J) are representative of at least three independent experiments. N.S., not significant, ${ }^{\star \star} P<0.01$, ${ }^{\star \star \star} P<0.001$ by two-tailed Student's $t$-test. The statistical significance of Normal-vs-Primary is 8.68299876444212E-11 (K). The statistical significance of Normal-vs-Gleason score 6 is 1.435520E-03; Normal-vs-Gleason score 7 is 3.24329999523698E-08; Normal-vs-Gleason score 8 is 7.61050000019914 E-07; Normal-vs-Gleason score 9 is 1.48140388844809E-11; Gleason score 6-vs-Gleason score 8 is 1.363590E-02; Gleason score 6-vs-Gleason score 9 is 2.406600 E-04; Gleason score 7-vs-Gleason score 9 is $3.778400 \mathrm{E}-03$ (L).

\section{DISCUSSION}

During miRNA biogenesis, the 5p strand of miRNA duplex is usually loaded into RISC and selected as dominant mature miRNA (27). Here, we found that miR-33b-3p was the dominant mature form in prostate cancer. miR-33b-3p were expressed over miR-33b$5 p$ in prostate cancer cell lines and tumor tissues. It is unclear how prostate cancer cells select miR-33b-3p as the dominant mature form in the current study. Recent study indicates that high level of the terminal uridylyl transferases TUT4 and TUT7 leads to selection of the $3 p$ strand instead of the $5 p$ strand of miR-324 (28).
In addition, target mRNA of miRNA also determines the arm selection of miRNA (18). Given that miR-33b-5p could be detected as a major mature form in other type of cancer tissues $(29,30)$, we deduced that miR-33b-3p selection in prostate cancer might depend on the prostate cell type.

Recently, miRNAs were implicated in cancer metastasis and emerged as "metastamir" or metastasis suppressor (31-33). miR$33 \mathrm{~b}-3 \mathrm{p}$ is recognized as an oncogenic or tumor suppressive molecule depending on different cell context. It targets the wellknown tumor suppressor CDKN1A (p21) and contributes to cisplatin-resistance in lung cancer (34). Overexpression of miR- 
TABLE 2 | Expression of potential target genes of miR-33b-3p in prostate cancer.

\begin{tabular}{lccc}
\hline Potential genes & \multicolumn{2}{c}{ TCGA samples } & Normal-vs-Primary tumor \\
\cline { 2 - 3 } & $\begin{array}{c}\text { Normal }(\mathbf{n}=\mathbf{5 2}) \\
\text { Median }\end{array}$ & $\begin{array}{c}\text { Primary tumor }(\mathbf{n}=\mathbf{4 9 7}) \\
\text { Median }\end{array}$ & Statistical significance \\
\hline PAFAH1B2 & 35.13 & 23.57 & $1.46 \mathrm{E}-06$ \\
FEZF1 & 0.00 & 0.00 & $2.52 \mathrm{E}-07$ \\
CLOCK & 5.12 & 3.26 & $5.60 \mathrm{E}-03$ \\
EPHA7 & 5.43 & 3.17 & $9.36 \mathrm{E}-04$ \\
AGO1 & 12.74 & 12.24 & $6.82 \mathrm{E}-02$ \\
BCL11B & 0.46 & 0.32 & $7.58 \mathrm{E}-02$ \\
GABBR2 & 0.05 & 0.03 & $5.05 \mathrm{E}-01$ \\
DOCK4 & $\mathbf{5 . 3 9}$ & $\mathbf{7 . 7 3}$ & $\mathbf{8 . 6 8 E - 1 1}$ \\
DLG2 & 3.12 & 2.08 & $5.60 \mathrm{E}-03$ \\
SYT1 & 5.80 & 1.97 & $1.81 \mathrm{E}-02$ \\
PLBD1 & 15.00 & 4.51 & $9.39 \mathrm{E}-11$ \\
FAT2 & 4.42 & 0.74 & $1.01 \mathrm{E}-07$ \\
UR11 & 68.03 & 69.63 & $4.62 \mathrm{E}-04$ \\
TOB1 & 144.84 & 123.62 & $8.26 \mathrm{E}-03$ \\
PRAMEF17 & 0.00 & 0.00 & $\mathrm{~N} / \mathrm{A}$
\end{tabular}

The data generated by TCGA Research Network (http://cancergenome. nih. gov/) has been used for UALCAN analysis (http://ualcan.path. uab.edu). Significance of difference estimated by Student's t-test.

N/A, not applicable. The most upregulated gene DOCK4 in prostatae tumor tissues was highlighted with bold.
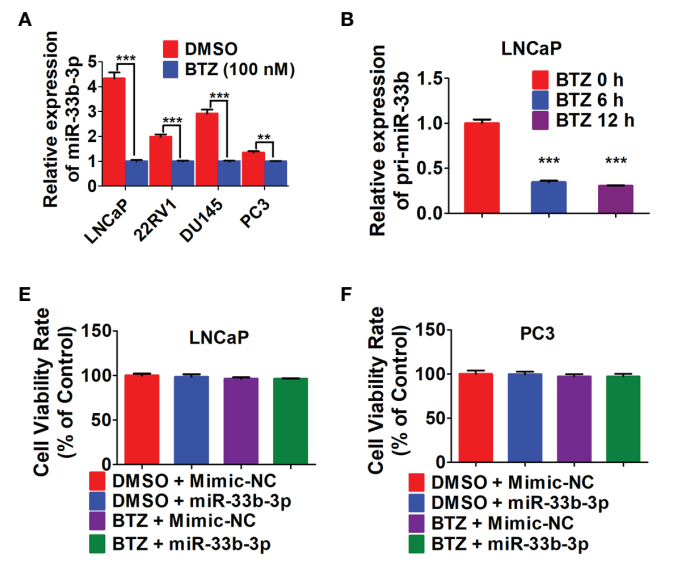

H
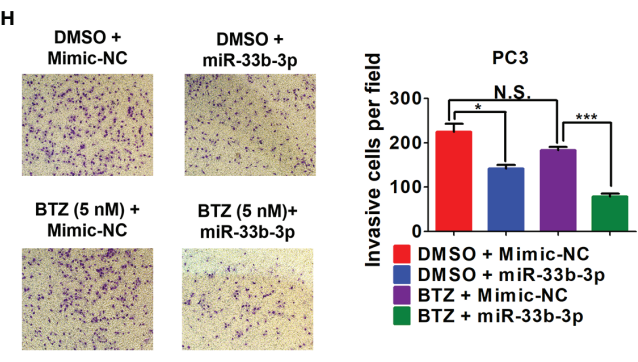

C

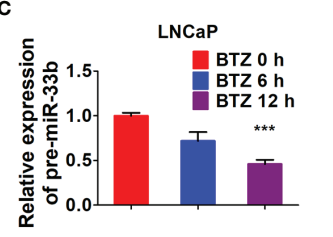

G
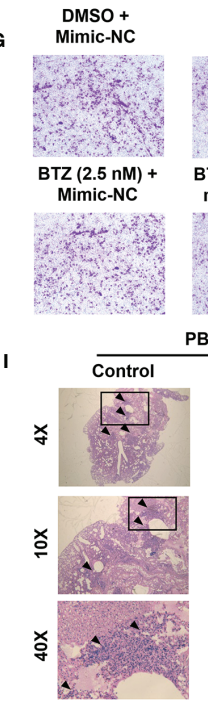

$4 / 4$

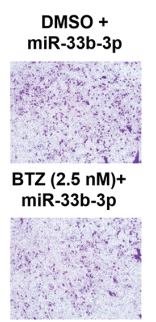

PBS miR-33b-3p
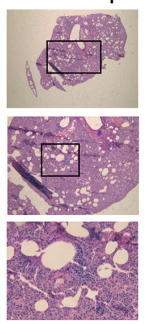

$1 / 4$
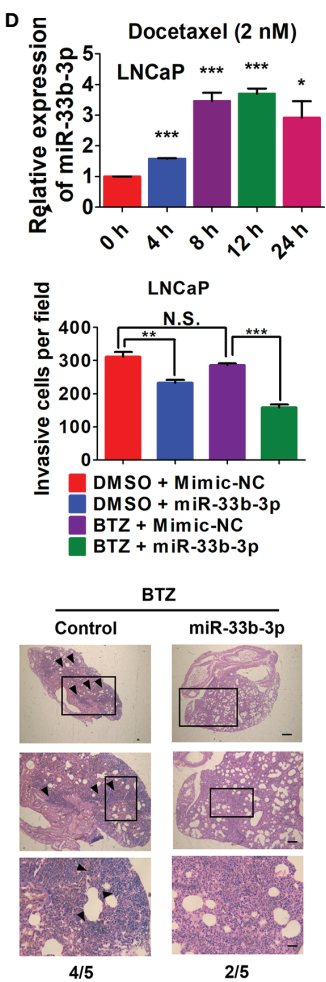

FIGURE 6 | Overexpression of miR-33b-3p enhances bortezomib effects. (A) RT-qPCR analysis of miR-33b-3p expression in prostate cancer cells post treatment with bortezomib (BTZ) (100 nM) for $24 \mathrm{~h}$. (B, C) RT-qPCR analysis the expression of pri- miR-33b and pre-miR-33b in LNCaP cells treated with BTZ (100 nM) for up to $12 \mathrm{~h}$. (D) Relative expression level of miR-33b-3p in LNCaP cells treated with Docetaxel (2 nM) for up to $24 \mathrm{~h}$. (E-H) miR-33b-3p overexpression and control cells were treated with or without BTZ (2.5 nM in LNCaP, $5 \mathrm{nM}$ in PC3 cells) for $48 \mathrm{~h}$, followed by flow cytometry (E, F) and Transwell assays (G, H). (I) H\&E staining in lung tissues. miR-33b-3p overexpression (miR-33b-3p) and control PC-3M-1E8-luciferase cells were injected into the mice through tail vein. After 30-days inoculation, each group was administrated with BTZ $(n=5)$ at a dose of $0.5 \mathrm{mg} / \mathrm{kg}$ body weight in $100 \mathrm{uL}$ or equal volume of PBS ( $\mathrm{n}=4$ ), respectively. Removed lungs from each group were stained by H\&E. The numbers of mice developed lung metastasis were indicated. Arrowheads indicate cancer metastasis foci. The scale bar represents $4 \times, 10 \mathrm{~mm} ; 10 \times, 5 \mathrm{~mm}$; and 40x, $1 \mathrm{~mm}$. Data (A-H) are representative of three independent experiments. N.S., not significant, ${ }^{\star} P<0.05,{ }^{\star \star} P<0.01$, ${ }^{* \star *} P<0.001$ by two-tailed Student's $t$-test. 
33b-3p enhanced cell proliferation and colony formation in gastric cancer (35), indicating the oncomir function of miR-33b-3p. On the other hand, downregulation of miR-33b-3p was found associated with aggressive phenotype of bladder cancer (22). In addition, decreased level of circulating miR-33b-3p was detected in patients with upper tract urothelial carcinoma in comparison with normal control group (36), supporting the tumor suppressive role of miR$33 \mathrm{~b}-3 \mathrm{p}$. In line with these observations, our results demonstrate that miR-33b-3p is a metastasis suppressor in prostate cancer. Low expression level of miR-33b-3p was detected in patients with prostate cancer and associated with their shortened survival period. Overexpression of miR-33b-3p suppressed whereas inhibition of miR-33b-3p promoted the migration and invasion abilities of cancer cells. Furthermore, in vivo study confirmed the anti-metastasis function of miR-33b-3p, showing that miR-33b-3p decreased the metastasis of prostate cancer cells to lymph nodes, lung, as well as liver. In this study, miR-33b-3p also inhibit the proliferation of prostate cancer cells. Our RNA-Seq data indicate that miR-33b-3p suppresses biological process such as angiogenesis and endothelial cell migration, suggesting that miR-33b-3p might regulate extensive pathways involved in prostate cancer cells homing and growth in the metastatic sites. We cannot eliminate the influence of these biological processes on metastasis, which is the limitation of our current study.

The current study demonstrates that miR-33b-3p inhibits metastasis by targeting DOCK4 in prostate cancer. Overexpression of DOCK 4 abolished the inhibition of miR-33b-3p on migration and invasion in prostate cancer cells. DOCK4 is a member of the DOCK180 family of guanine nucleotide exchange factor. It was first identified as a Rap GTPase activator that can enhance the formation of adherent junctions between cells, mutations of which was found to confer the loss of cell junction and an invasive phenotype in cancer (37). However, subsequent studies indicate that DOCK4 might promote cell invasion rather than cell-cell adhesion. Overexpression of DOCK4 in placental cytotrophoblasts increased the invasiveness of these cells (38). Upregulation of DOCK4 was found associated with metastases of breast and lung cancer $(39,40)$. In this study, overexpression of wild type of DOCK4 promoted the migration and invasion of prostate cancer cells, supporting its function to mediate metastasis in prostate cancer.

The proteasome is a validated target for cancer therapy. Proteasome inhibition represents a successive strategy for the treatment of multiple myeloma. However, proteasome inhibitors failed to benefit to patients with solid tumors for some reasons (41). The current study suggests that downregulation of miR$33 \mathrm{~b}-3 \mathrm{p}$ is one of the reasons resulting in bortezomib limited efficacy in prostate cancer. Under non-cytotoxic doses, bortezomib had no effect on inhibition of cell invasion, while

\section{REFERENCES}

1. Siegel RL, Miller KD, Fuchs HE, Jemal A. Cancer Statistics, 2021. CA Cancer J Clin (2021) 71:7-33. doi: 10.3322/caac.21654

2. Jang A, Sartor O, Barata PC, Paller CJ. Therapeutic Potential of PARP Inhibitors in the Treatment of Metastatic Castration-Resistant Prostate Cancer. Cancers (Basel) (2020) 12:3467. doi: 10.3390/cancers12113467 overexpression of miR-33b-3p could improve bortezomib suppression on migration and invasion. Therefore, the current study suggests that overcoming miR-33b-3p downregulation might increase bortezomib efficacy against prostate cancer.

In summary, our results demonstrate the anti-tumor function of miR-33b-3p in prostate cancer. It suppresses the invasion and migration of prostate cancer cells by targeting DOCK4. Proteasome inhibitor bortezomib leads to insufficient inhibition on cell invasion due to miR-33b-3p downregulation, which can be overcome by overexpression of miR-33b-3p in prostate cancer.

\section{DATA AVAILABILITY STATEMENT}

The datasets presented in this study can be found in online repositories. The names of the repository/repositories and accession number(s) can be found below: NCBI GEO repository, GSE183428.

\section{ETHICS STATEMENT}

The animal study was reviewed and approved by Animal Care and Use Committee, Harbin Institute of Technology

\section{AUTHOR CONTRIBUTIONS}

$\mathrm{HuY}$ and YM conceived the project, analyzed the data, and wrote the paper. YM and KL designed and performed the experiments. $\mathrm{ML}$ and SS contributed to the in vivo experiments. ZZ, ML, HuY, $\mathrm{HW}, \mathrm{XH}$ and XL helped other experiments. All authors contributed to the article and approved the submitted version.

\section{FUNDING}

This work was supported by National Natural Science Foundation of China (81872439), Natural Science Foundation of Heilongjiang Province (LH2020H072) and Innovation Research Project of Harbin (2017RAQXJ182).

\section{SUPPLEMENTARY MATERIAL}

The Supplementary Material for this article can be found online at: https://www.frontiersin.org/articles/10.3389/fonc.2021.740452/ full\#supplementary-material

3. Di Lorenzo G, Buonerba C, Autorino R, De Placido SN, Sternberg C. Castration-Resistant Prostate Cancer: Current and Emerging Treatment Strategies. Drugs (2010) 70:983-1000. doi: 10.2165/10898600-00000000000000

4. Yang H, Zonder JA, Dou QP. Clinical Development of Novel Proteasome Inhibitors for Cancer Treatment. Expert Opin Investig Drugs (2009) 18:95771. doi: $10.1517 / 13543780903002074$ 
5. Hainsworth JD, Meluch AA, Spigel DR, Barton J, Simons L, Meng C, et al. Weekly Docetaxel and Bortezomib as First-Line Treatment for Patients With HormoneRefractory Prostate Cancer: A Minnie Pearl Cancer Research Network Phase II Trial. Clin Genitourin Cancer (2007) 5:278-83. doi: 10.3816/CGC.2007.n.004

6. Rescigno P, Buonerba C, Bellmunt J, Sonpavde G, De Placido S, Di Lorenzo G. New Perspectives in the Therapy of Castration Resistant Prostate Cancer. Curr Drug Targets (2012) 13:1676-86. doi: 10.2174/138945012803529956

7. Fang L, Li H, Wang L, Hu J, Jin TR, Wang JP, et al. MicroRNA-17-5p Promotes Chemotherapeutic Drug Resistance and Tumour Metastasis of Colorectal Cancer by Repressing PTEN Expression. Oncotarget (2014) 5:2974-87. doi: 10.18632/oncotarget.1614

8. Rokavec M, Öner MG, Li H, Jackstadt R, Jiang L, Lodygin D, et al. IL-6r/STAT3/ miR-34a Feedback Loop Promotes EMT-Mediated Colorectal Cancer Invasion and Metastasis. J Clin Invest (2014) 124:1853-67. doi: 10.1172/JCI73531

9. Fujita Y, Kojima K, Ohhashi R, Hamada N, Nozawa Y, Kitamoto A, et al. MiR-148a Attenuates Paclitaxel Resistance of Hormone-Refractory, DrugResistant Prostate Cancer PC3 Cells by Regulating MSK1 Expression. J Biol Chem (2010) 285:19076-84. doi: 10.1074/jbc.M109.079525

10. Choi N, Park J, Lee JS, Yoe J, Park GY, Kim E, et al. miR-93/miR-106b/miR375-CIC-CRABP1: A Novel Regulatory Axis in Prostate Cancer Progression. Oncotarget (2015) 6:23533-47. doi: 10.18632/oncotarget.4372

11. Wang Y, Lieberman R, Pan J, Zhang Q, Du MJ, Zhang P, et al. miR-375 Induces Docetaxel Resistance in Prostate Cancer by Targeting SEC23A and YAP1. Mol Cancer (2016) 15:70. doi: 10.1186/s12943-016-0556-9

12. Manier S, Liu CJ, Avet-Loiseau H, Park J, Shi JT, Campigotto F, et al. Prognostic Role of Circulating Exosomal miRNAs in Multiple Myeloma. Blood (2017) 129:2429-36. doi: 10.1182/blood-2016-09-742296

13. Łuczkowska K, Rogińska D, Ulańczyk Z, Paczkowska E, Schmidt CA, Machaliński B. Molecular Mechanisms of Bortezomib Action: Novel Evidence for the miRNA-mRNA Interaction Involvement. Int $J \mathrm{Mol} \mathrm{Sci}$ (2020) 21:350. doi: 10.3390/ijms 21010350

14. Wang H, Li K, Mei Y, Huang XM, Li ZL, Yang QZ, et al. Sp1 Suppresses miR-3178 to Promote the Metastasis Invasion Cascade via Upregulation of TRIOBP. Mol Ther Nucleic Acids (2018) 12:1-11. doi: 10.1016/j.omtn.2018.04.008

15. Hao M, Zang M, Wendlandt E, Xu Y, An G, Gong DS, et al. Low Serum miR19a Expression as a Novel Poor Prognostic Indicator in Multiple Myeloma. Int J Cancer (2015) 136:1835-44. doi: 10.1002/ijc.29199

16. Tian Z, Zhao JJ, Tai YT, Amin SB, Hu YG, Berger AJ, et al. Investigational Agent MLN9708/2238 Targets Tumor-Suppressor Mir33b in MM Cells. Blood (2012) 120:3958-67. doi: 10.1182/blood-2012-01-401794

17. Guo L, Yu J, Yu H, Zhao Y, Chen SJ, Xu CQ, et al. Evolutionary and Expression Analysis of miR-\#-5p and miR-\#-3p at the miRNAs/isomiRs Levels. BioMed Res Int (2015) 2015:168358. doi: 10.1155/2015/168358

18. Medley JC, Panzade G, Zinovyeva AY. microRNA Strand Selection: Unwinding the Rules. Wiley Interdiscip Rev RNA (2021) 12:e1627. doi: 10.1002/wrna.1627

19. Zhang Z, Pi J, Zou D, Wang XS, Xu JY, Yu S, et al. microRNA Arm-Imbalance in Part From Complementary Targets Mediated Decay Promotes Gastric Cancer Progression. Nat Commun (2019) 10:4397. doi: 10.1038/s41467-019-12292-5

20. Zhao M, Qi M, Li X, Hu J, Zhang J, Jiao M, et al. CUL4B/miR-33b/C-MYC Axis Promotes Prostate Cancer Progression. Prostate (2019) 79:480-8. doi: 10.1002/pros.23754

21. Gao L, Xiong DD, He RQ, Lai ZF, Liu LM, Huang ZG, et al. Identifying TFmiRNA-mRNA Regulatory Modules in Nitidine Chloride Treated HCC Xenograft of Nude Mice. Am J Transl Res (2019) 11:7503-22.

22. Inamoto T, Uehara H, Akao Y, Ibuki N, Komura K, Takahara K, et al. A Panel of MicroRNA Signature as a Tool for Predicting Survival of Patients With Urothelial Carcinoma of the Bladder. Dis Markers (2018) 2018:5468672. doi: $10.1155 / 2018 / 5468672$

23. Pfaffl MW. A New Mathematical Model for Relative Quantification in RealTime RT-PCR. Nucleic Acids Res (2001) 29:e45. doi: 10.1093/nar/29.9.e45

24. Gao Y, Zhang Z, Li K, Gong L, Yang Q, Huang X, et al. Linc-DYNC2H1-4 Promotes EMT and CSC Phenotypes by Acting as a Sponge of miR-145 in Pancreatic Cancer Cells. Cell Death Dis (2017) 8:e2924. doi: 10.1038/cddis.2017.311

25. Guo J, Huang X, Wang H, Yang HJ. Celastrol Induces Autophagy by Targeting AR/miR-101 in Prostate Cancer Cells. PloS One (2015) 10: e0140745. doi: 10.1371/journal.pone.0140745
26. Liu Y, Zheng J, Fang W, You J, Wang J, Cui X, et al. Isolation and Characterization of Human Prostate Cancer Cell Subclones With Different Metastatic Potential. Zhonghua Bing Li Xue Za Zhi (1999) 28:361-4.

27. Bartel DP. MicroRNAs: Genomics, Biogenesis, Mechanism, and Function. Cell (2004) 116:281-97. doi: 10.1016/S0092-8674(04)00045-5

28. Kim H, Kim J, Yu S, Lee YY, Park J, Choi RJ, et al. A Mechanism for microRNA Arm Switching Regulated by Uridylation. Mol Cell (2020) 78:1224-36.e5. doi: 10.1016/j.molcel.2020.04.030

29. Li F, Hao M, Feng X, Zang MR, Qin Y, Yi SH, et al. Downregulated miR-33b is a Novel Predictor Associated With Disease Progression and Poor Prognosis in Multiple Myeloma. Leuk Res (2015) 39:793-9. doi: 10.1016/j.leukres.2015.04.010

30. Wang H, Lin X, Liu E, Jian ZY, Ou YL. MicroRNA-33b Regulates Hepatocellular Carcinoma Cell Proliferation, Apoptosis, and Mobility via Targeting Fli-1-Mediated Notch1 Pathway. J Cell Physiol (2020) 235:7635-44. doi: 10.1002/jcp.29673

31. Kim J, Yao F, Xiao Z, Sun Y, Ma L. MicroRNAs and Metastasis: Small RNAs Play Big Roles. Cancer Metastasis Rev (2018) 37:5-15. doi: 10.1007/s10555017-9712-y

32. Hurst DR, Edmonds MD, Welch DR. Metastamir: The Field of MetastasisRegulatory microRNA is Spreading. Cancer Res (2009) 69:7495-8. doi: 10.1158/0008-5472.CAN-09-2111

33. Edmonds MD, Hurst DR, Welch DR. Linking Metastasis Suppression With metastamiR Regulation. Cell Cycle (2009) 8:2673-5. doi: 10.4161/cc.8.17.9303

34. Xu S, Huang H, Chen YN, Deng YT, Zhang B, Xiong XD, et al. DNA Damage Responsive miR-33b-3p Promoted Lung Cancer Cells Survival and Cisplatin Resistance by Targeting P21(WAF1/CIP1). Cell Cycle (2016) 15:2920-30. doi: 10.1080/15384101.2016.1224043

35. Sexton R, Mahdi Z, Chaudhury R, Beydoun R, Aboukameel A, Khan HY, et al. Targeting Nuclear Exporter Protein XPO1/CRM1 in Gastric Cancer. Int J Mol Sci (2019) 20:4826. doi: 10.3390/ijms20194826

36. Tao J, Yang X, Li P, Wei JF, Deng XH, Cheng YD, et al. Identification of Circulating microRNA Signatures for Upper Tract Urothelial Carcinoma Detection. Mol Med Rep (2015) 12:6752-60. doi: 10.3892/mmr.2015.4257

37. Yajnik V, Paulding C, Sordella R, McClatchey AI, Saito M, Wahrer DCR, et al. DOCK4, a GTPase Activator, is Disrupted During Tumorigenesis. Cell (2003) 112:673-84. doi: 10.1016/S0092-8674(03)00155-7

38. McNally L, Zhou Y, Robinson JF, Zhao GF, Chen LM, Chen H, et al. UpRegulated Cytotrophoblast DOCK4 Contributes to Over-Invasion in Placenta Accreta Spectrum. Proc Natl Acad Sci USA (2020) 117:15852-61. doi: 10.1073/ pnas. 1920776117

39. Yu JR, Tai Y, Jin Y, Hammell MC, Wilkinson JE, Roe JS, et al. TGF- $\beta /$ Smad Signaling Through DOCK4 Facilitates Lung Adenocarcinoma Metastasis. Genes Dev (2015) 29:250-61. doi: 10.1101/gad.248963.114

40. Westbrook JA, Wood SL, Cairns DA, McMahon K, Gahlaut R, Thygesen H, et al. Identification and Validation of DOCK4 as a Potential Biomarker for Risk of Bone Metastasis Development in Patients With Early Breast Cancer. J Pathol (2019) 247:381-91. doi: 10.1002/path.5197

41. Manasanch EE, Orlowski RZ. Proteasome Inhibitors in Cancer Therapy. Nat Rev Clin Oncol (2017) 14:417-33. doi: 10.1038/nrclinonc.2016.206

Conflict of Interest: The authors declare that the research was conducted in the absence of any commercial or financial relationships that could be construed as a potential conflict of interest.

Publisher's Note: All claims expressed in this article are solely those of the authors and do not necessarily represent those of their affiliated organizations, or those of the publisher, the editors and the reviewers. Any product that may be evaluated in this article, or claim that may be made by its manufacturer, is not guaranteed or endorsed by the publisher.

Copyright $\odot 2021$ Mei, Li, Zhang, Li, Yang, Wang, Huang, Li, Shi and Yang. This is an open-access article distributed under the terms of the Creative Commons Attribution License (CC BY). The use, distribution or reproduction in other forums is permitted, provided the original author(s) and the copyright owner(s) are credited and that the original publication in this journal is cited, in accordance with accepted academic practice. No use, distribution or reproduction is permitted which does not comply with these terms. 